\title{
Smoking advice for periodontitis patients
}

\author{
Smoking cessation advice for patients with chronic periodontitis \\ H. A. Nasry, P. M. Preshaw, F. Stacey, L. Heasman, M. Swan and P. A. Heasman Br Dent J 2006; \\ 200: 272-275
}

\begin{abstract}
Background
There are limited data on the utility of dental professionals in providing smoking cessation counselling in the UK.

Objectives

The purpose of this study was to determine quit rates for smokers with chronic periodontitis who were referred to a dental hospital for treatment.

Materials and methods

Forty-nine subjects with chronic periodontitis who smoked cigarettes were recruited. All subjects received periodontal treatment and smoking cessation advice as part of an individual, patient-based programme provided by dental hygienists trained in smoking cessation counselling. Smoking cessation interventions included counselling (all patients), and some patients also received nicotine replacement therapy (NRT) and/or Zyban medication. Smoking cessation advice was given at each visit at which periodontal treatment was undertaken (typically four to six visits) over a period of 10-12 weeks. Smoking cessation advice was also given monthly during the programme of supportive periodontal care over the following nine months. Smoking status was recorded at three, six and 12 months and was confirmed with carbon monoxide (CO) monitors and salivary cotinine assays.

Results

Forty-one per cent, 33\%, 29\% and 25\% of patients had stopped smoking at week four, months three, six and 12, respectively. Gender, age, the presence of another smoker in the household, and baseline smoking status (determined using subject-reported pack years of smoking) were not significant predictors of quit success $(P>0.05)$. Baseline $C O$ levels were significantly associated with quit success, however, and were significantly higher in those subjects who continued to smoke compared to those subjects who were quitters at week four, month three and month six $(P<0.05)$.

Conclusion

Success rates in quitting smoking following smoking cessation advice given as part of a periodontal treatment compared very favourably to national quit rates achieved in specialist smoking cessation clinics. The dental profession has a crucial role to play in smoking cessation counselling, particularly for patients with chronic periodontitis.
\end{abstract}

\section{IN BRIEF}

- Twelve million adults smoke in the UK, and smoking is responsible for 114,000 deaths each year.

- Carbon monoxide levels may be a good indicator of the degree of addiction to nicotine.

- The dental profession has a crucial role to play in smoking cessation counselling, particularly for patients with chronic periodontitis.

- This study shows that there is great potential for smoking cessation intervention in clinic settings.

\section{COMMENT}

Smoking is recognised as a major cause of both morbidity and mortality. The effects of the habit on oral health, particularly regarding oral and pharyngeal cancers and periodontal diseases, are also well documented making assisting patients in quitting smoking a priority. The aim of this study was to evaluate the role of hygienists trained in smoking cessation counselling in providing smoking cessation advice within a secondary care environment to a cohort of patients with periodontal disease.

Quit rates were assessed at four time points (four weeks and three, six and 12 months) using three different methods: selfreporting, carbon monoxide (CO) content in expired air and salivary cotinine concentration. The results show quit rates of $41 \%$, $33 \%, 29 \%$ and $25 \%$ respectively at these times. These findings are very impressive compared with other studies published in both the dental and non-dental literature and highlight the great potential for success with this approach. The authors attribute the excellent results gained to the high quality of smoking cessation training received by the dental hygienists involved and the frequent contact and close working relationships developed with the patients and recognise both that the number of study participants is relatively small and that the research needs to broadened out into the primary care setting. However, the major take home message should be the encouragement it provides for the routine provision of smoking cessation advice by all the members of the dental team.

Another intriguing result is the finding that base line $\mathrm{CO}$ readings were significantly lower in patients who were quitters at four weeks, three months and six months than readings from nonquitters. The authors postulate that $\mathrm{CO}$ levels may thus be a good indicator of the degree of addiction to nicotine. Conversely, salivary cotinine scores were significantly lower in quitters compared to non-quitters only at month 12 suggesting that this method may be less useful in the prediction of quitting outcome. Success in quitting was not related to gender, age, presence of another smoker in the household, base-line smoking status (determined as self-reported pack years of smoking) or quit method used though further research is clearly indicated to confirm these findings.

In short, this excellent paper provides evidence to the dental team that provision of smoking cessation advice is worthwhile and the message should be taken on board if both disease prevention and treatment efficacy are to be maximised.

A. Gilbert, Senior Lecturer in Periodontology, University of Dundee. doi: 10.1038/sj.bdj.4813308 